\title{
Un élément de réponse inverse aux hormones thyroïdiennes
}

L'effet des hormones thyroïdiennes est relayé par les protéines c-ErbA, membres de la super-famille des récepteurs nucléaires. Ces récepteurs sont des facteurs de transcription qui se fixent à leur élément de réponse sur l'AI)N, principalement sous la forme d'hétérodimères avec un membre de la famille $\operatorname{RXR}(\mathrm{m} / \mathrm{s}$ $n^{\circ}$ 3, vol. $8, p$. 283). I.es éléments de réponse aux hormones thyroïdiennes, ou TRE, sont souvent composés de deux hémi-sites hexamériques, directement répétés et espacés de quatre bases $\left(\mathrm{m} / \mathrm{s} n^{\circ} 8\right.$, vol. 7 , p. 863). ('ependant, d'autres TRE, moins fréquents, sont des palindromes dont les hémi-sites sont très proches de ceux des ERE (estrogen response element), du type A/GGGT(;/CA. I.es TRE sont normalement reconnus par les protéines c-ErbA liées ou non aux hormones thyroïdiennes. Cependant, cErbA non lié se comporte comme un inhibiteur, alors qu'il devient un activateur lorsqu'il est complexé à l'hormone. Le produit de l'oncogène verbA du virus de l'érythroblastose aviaire est la contrepartie virale du gène cellulaire cerbA mais a subi des modifications le rendant insensible à l'activation par les hormones thyroïdiennes: de ce fait, la protéine v-ErbA est un inhibiteur compétitif de l'effet des hormones thyroïdiennes. Une autre action de ces hormones est de stimuler le pouvoir inhibiteur de c-ErbA sur le facteur de transcription AP1, facteur relayant l'action de nombreux stimuli proli$\mathrm{m} / \mathrm{s} n^{\circ} 3$ vol. 10, mars 94 fératifs. En revanche, v-ErbA empêche cette inhibition d'APl dépendante des hormones $\left(\mathrm{m} / \mathrm{s} n^{\circ} 2\right.$, vol. 8 , p. 156). F. Saatcoglu, du laboratoire de M. Karin, Ia Jolla (CA, USA) décrit maintenant un tout nouveau type de TRE dont les effets sont tout à fait inversés par rapport aux TRE classiques [1]. Cet élément, présent dans le I.TR (long terminal repeat) du virus du sarcome de Rous, a une structure singulière: il est formé par une répétition inversée de l'hexamère AAGGCA, les deux demi-sites étant séparés de six bases. Ia séquence des hémi-sites aussi bien que la longueur de l'espace entre eux sont fondamentales pour l'effet biologique singulier de ce TRE. En effet, cet élément d'ADN lie les protéines c-ErbA et v-ErbA mais, contrairement aux TRE classiques, relaie une activation transcriptionnelle par ces facteurs indépendante de la liaison aux hormones thyroidiennes (au moins pour l'isoforme $c-E r b A \alpha$, la protéine c-ErbA $\beta$ étant inactive). La liaison à l'hormone, évidemment inactive sur v-ErbA, supprime l'effet activateur de c-ErbA, tout à l'inverse de ce qui était décrit jusqu'à présent. L'interprétation de ces résultats ne peut être que spéculative. Il est probable que la structure particulière de ce TRE imprime au récepteur fixé (homodimères cErbA ou v-ErbA, ou hétérodimères impliquant également RXR) une conformation spéciale démasquant un domaine d'activation transcriptionnelle. Ce domaine semble être porté par l'extrémité aminoterminale des molécules, la partie carboxy-terminale, qui fixe le ligand, n'étant pas indispensable à la transactivation en l'absence d'hormone. En revanche, les molécules possèdant cette extrémité carboxyterminale et, ainsi, susceptibles de fixer l'hormone, subiraient, en présence de l'hormone, une nouvelle trans-conformation aboutissant au masquage du domaine transactivateur amino-terminal. ('es phénomènes seraient assez exactement inverses de ceux survenant lorsque cErbA se fixe à un TRE ordinaire. L'importance biologique de ce système ne peut être encore précisée mais les auteurs de l'article commenté ici indiquent qu'une recherche dans les banques de données a permis d'identifier, en amont de nombreux gènes, des séquences ayant des structures similaires à celles du “TRE inverse" du I.TR du virus de Rous.

A.K.

1. Saatcoglu F, Deng T, Karin M. A novel cis element mediating ligand-independent activation by c-ErbA: implications for hormonal regulation. Cell 1993; 75: 1065-105. 